\title{
La intervención intensiva para la cesación tabáquica en pacientes internados no demostró ser mejor que el soporte habitual
}

Systematic identification and treatment of smokers in a secondary care setting didn't prove to be more effective than usual care

Murray RL. BMJ 2013;347:f4004.

\section{Objetivos}

Determinar si la identificación sistemática y la oferta de soporte por especialistas en cesación tabáquica a los pacientes fumadores internados es más efectiva que el soporte que se brinda habitualmente por el médico tratante.

\section{Diseño}

Ensayo clínico, controlado, aleatorizado y abierto.

\section{Lugar y pacientes}

Hospital de Nottingham, Reino Unido. Se incluyeron adultos internados, fumadores actuales o que fumaron en las últimas cuatro semanas. Se utilizaron los sectores de internación como unidad de aleatorización, aplicando la misma de manera secuencial.

\section{Intervención}

La intervención consistía en la detección sistemática, provisión de soporte conductual y farmacoterapia durante la internación a todos los fumadores y ex-fumadores recientes por especialistas en cesación tabáquica. Se hacía además seguimiento y referencia a centros comunitarios luego del alta. El soporte habitual consistía en la oferta de soporte para la cesación tabáquica por el médico tratante. Fueron incluidos 493 pacientes en el estudio, de los cuales 264 fueron asignados al grupo de intervención y 229 al grupo de soporte habitual.

\section{Medición de resultados principales}

El resultado principal fue la cesación tabáquica a las cuatro semanas, constatado por la exhalación de monóxido de carbono. El análisis de los resultados fue por intención de tratar.

\section{Resultados principales}

Se resumen en la tabla 1.

Tabla 1: chance de éxito al comparar una intervención intensiva especializada versus cuidado usual de cesación tabáquica en pacientes internados.

\begin{tabular}{c|c|c|c|c}
\hline Resultado & Grupo intervención & Grupo soporte habitual & OR (IC95\%) & $\mathbf{p}$ \\
\hline Cesación a las cuatro semanas & $38 \%$ & $17 \%$ & $2,10(0,96$ a 4,61) & 0,06 \\
\hline
\end{tabular}

OR: Odds Ratio

\section{Conclusión de los autores}

La cesación tabáquica a las cuatro semanas no mostró ser mejor en el grupo de intervención ya que los resultados no fueron estadísticamente significativos.
Fuente de financiamiento: Los autores refieren no haber recibido ningún tipo ayuda por parte de organizaciones, ni haber tenido ninguna relación financiera con las mismas en los últimos tres años.

\section{Comentario}

La identificación de los pacientes fumadores en la internación y la consecuente oferta de soporte para la cesación tabáquica forma parte de las recomendaciones de las guías de Estados Unidos y el Reino Unido hace ya quince años. Existe evidencia que demuestra que la intervención intensiva en los pacientes fumadores internados y su posterior seguimiento al alta incrementa la tasa de cesación tabáquica y que el efecto de la misma es independiente del motivo de ingreso ${ }^{2}$. Sin embargo, la mayoría de los hospitales no adopta estas medidas sistemáticamente, delegando al equipo tratante la decisión de ofrecerlas.

Este trabajo intenta demostrar mediante un ensayo clínico que este tipo de intervención es más efectiva que lo que se realiza habitualmente. No obstante, no llega a obtener resultados significativos. Esto pudo haber ocurrido, por un menor número de participantes por sectores que lo esperado. A su vez, presenta algunas limitaciones, como haber estudiado pacientes de un único hospital, y el hecho de separar a los participantes por sectores de internación. Si bien esto disminuye la posibilidad de contaminación entre grupos, también podría atentar contra la validez interna del estudio.

\section{Conclusiones del comentador}

La intervención intensiva en los pacientes fumadores internados y su posterior seguimiento al alta no incrementó la tasa de cesación tabáquica. Siendo el consejo breve una intervención altamente costo-efectiva, es recomendable ofrecerlo a todos los pacientes fumadores en cualquier nivel de atención.

Paula Riganti [ Medica de Familia, Servicio de Medicina Familiar del Hospital Italiano de Buenos Aires. paula.riganti@hospitalitaliano.org.ar ]

Riganti P. La intervención intensiva para la cesación tabáquica en pacientes internados no demostró ser mejor que el soporte habitual. Evid Act Práct Ambul. 2014, 17(2). Abr-Jun. 55. Comentado de: Murray RL, Marsh J, Leonardi-Bee J, Jayes L, Britton J. Systematic identification and treatment of smokers by hospital based cessation practitioners in a secondary care setting: cluster randomised controlled trial. BMJ 2013;347:f4004 doi: 10.1136/bmj.f4004. PMID: 23836616.

\section{Referencias}

1. Lewis KE, Durgan L, Edwards VM, et al. Can smokers switch from a hospital-based to a community-based stop smoking service? An open-label, randomized trial comparing three referral schemes. Nicotine Tob Res 2009;11:756-64.

2. Rigotti NA, Clair C, Munafo MR, Stead LF. Interventions for smoking cessation in hospitalised patients. Cochrane Database Syst Rev 2012;5:CD001837. 